\title{
PROPAGATION, REFLECTION, AND DIFFRACTION OF SINGULARITIES OF SOLUTIONS TO WAVE EQUATIONS
}

\author{
BY MICHAEL E. TAYLOR ${ }^{1}$
}

\section{Contents}

0. Introduction

1. Fourier integral operators and propagation of singularities

2. Reflection of singularities

3. Grazing rays and diffraction

4. Diffraction theory and scattering theory

0. Introduction. This paper will survey recent progress in understanding the propagation of singularities of solutions to linear partial differential equations $P u=f$, particularly hyperbolic equations, such as the wave equation $\left(\partial^{2} / \partial t^{2}\right.$ $-\Delta) u=f$. Theorems describing this behavior, for general initial data, probably began with Lax [21] and Courant and Lax [6], although work on the problem dates back further. The method of analysis, known as geometrical optics, was used by Sommerfeld and Runge [44] and Birkhoff [2] in an effort to construct approximate solutions to the wave equation. This method was forged into a powerful tool, the theory of Fourier integral operators, by Hörmander [15], [16] and applied to get very general global results on propagation of singularities in [16] and [8].

In order to give a precise statement of Hörmander's theorem on propagation of singularities, we need to define the wave front set of a distribution, denoted WF( $u)$, where $u \in \mathscr{Q}^{\prime}(\Omega)$ is a distribution on some domain $\Omega \subset \mathbf{R}^{n}$. WF $(u)$ was introduced by Hörmander [15], based on Sato's notion of S. S. $u$ [42]. WF $(u)$ will be a subset of $T^{*}(\Omega) \approx \Omega \times \mathbf{R}^{n}$. One way to give the definition is to say $\left(x_{0}, \xi_{0}\right) \notin \mathrm{WF}(u)$ provided there is a $\varphi \in C_{0}^{\infty}(\Omega)$, $\varphi=1$ near $x_{0}$, such that $(\varphi u)^{\wedge}(\xi)$ is rapidly decreasing as $|\xi| \rightarrow \infty$ for $\xi$ in some open cone $\Gamma$ containing $\xi_{0}$. An equivalent definition, using pseudo differential operators, will be given in $\$ 1$. It turns out that the projection $T^{*}(\Omega) \rightarrow \Omega$ maps $\mathrm{WF}(u)$ onto the singular support of $u$ (sing supp $u$ ), so $\mathrm{WF}(u)$ provides finer information than sing supp $u$.

Now suppose $P u=f$ in $\Omega$. We suppose $P$ is a differential operator, or more generally a pseudo differential operator of order $m$, whose principal symbol $p_{m}(x, \xi)$, homogeneous of degree $m$ in $\xi$, is real valued. Let $q(x, \xi)=$ $|\xi|^{1-m_{p}}(x, \xi)$, and consider the Hamiltonian vector field on $T^{*}(\Omega)$ :

$$
H_{q}=\sum_{j=1}^{n}\left(\frac{\partial q}{\partial x_{j}} \frac{\partial}{\partial \xi_{j}}-\frac{\partial q}{\partial \xi_{j}} \frac{\partial}{\partial x_{j}}\right)
$$

An Invited Address presented at the Annual Meeting of the American Mathematical Society in Atlanta, Georgia, on January 6, 1978; received by the editors August 29, 1977.

AMS (MOS) subject classifications (1970). Primary 35L05, 35L15, 35L20.

'Work supported in part by NSF grant MCS77-03634. 
THEOREM (HÖRMANDER). WF $(u) \backslash \mathrm{WF}(f)$ is contained in char $p_{m}=\{(x, \xi)$ : $\left.p_{m}(x, \xi)=0\right\}$ and is invariant under the Hamiltonian flow generated by $H_{q}$ in $T^{*}(\Omega) \backslash \mathrm{WF}(f)$.

This result says nothing about the reflection of singularities of $u$ at the boundary $\partial \Omega$, which we now suppose to be smooth. If $P$ is a scalar differential operator of order $m$, and $\left(y_{0}, \zeta_{0}\right) \in T^{*}(\partial \Omega)$, there will be $k$ points $\left(y_{0}, \zeta_{j}\right) \in T^{*}(\Omega)$ lying over $\left(y_{0}, \zeta_{0}\right)(0<k \leqslant m)$ (i.e., $\left(y_{0}, \zeta_{0}\right)=\kappa^{*}\left(y_{0}, \zeta_{j}\right)$ where $\kappa: \partial \Omega \rightarrow \Omega$ ) which belong to char $P$, assuming $\partial \Omega$ is noncharacteristic. If we denote by $\gamma_{j}$ null bicharacteristic strips passing through $\left(y_{0}, \xi_{j}\right)$ (i.e., integral curves of $H_{q}$ ), and if we divide this set of rays into two groups, say $\gamma_{1}, \ldots, \gamma_{j}$, and $\gamma_{j+1}, \ldots, \gamma_{k}$, we will say these two groups of rays are related by reflection. If $u$, solving $P u=f$, satisfies certain boundary conditions, say $B u=g$ on $\partial \Omega$, one wants to know when smoothness of $u$ along $\gamma_{1}, \ldots, \gamma_{j}$ implies smoothness along the reflected rays $\gamma_{j+1}, \ldots, \gamma_{k}$. The simplest case to treat is when all the rays $\gamma_{1}, \ldots, \gamma_{k}$ are transversal to $\partial \Omega$. In such a case approximate solutions to $P u=f, B u=g$ can be computed using Fourier integral operators. Lax and Nirenberg [36] and also Chazarain [5] treated the Dirichlet problem, and general boundary value problems were treated by Majda and Osher [26] for scalar equations and by Taylor [48] for systems. It turns out that a Lopatinsky condition, reminiscent of the condition for regularity of an elliptic boundary value problem, leads to such reflection of singularities phenomena. This result is described in $\$ 2$. We should mention that certain boundary value problems that occur naturally, for example in linear elasticity, do not satisfy this Lopatinsky condition, and more complicated phenomena, such as Rayleigh waves, occur; this is also discussed in $\$ 2$.

The grazing ray problem, solved by Taylor [49] and Melrose [30], deals with the propagation of singularities along rays which hit $\partial \Omega$ tangentially, locally staying inside $\Omega$ near the point of contact, which is of precisely second order. This work leads, for example, to a complete analysis of the singularities of solutions to the wave equation $\left(\partial^{2} / \partial t^{2}-\Delta\right) u=0$ on the exterior of a smooth convex obstacle $K$ in $\mathbf{R}^{n}$, with for example Dirichlet or Neumann conditions on $\partial K$. In the case of the Dirichlet problem, important progress had been made by Ludwig [24] and by Morawetz and Ludwig [35]. The construction of approximate solutions needed to treat the grazing ray problem involves a class of operators more complicated than Fourier integral operators and is described in \$3. It was only with the solution of the grazing ray problem that tools became available to give rigorous mathematical treatments of a number of problems of classical scattering theory involving a convex obstacle, such as the analysis of the asymptotic behavior of the scattering matrix and the justification of a number of results that previously had been obtained using Kirchoff's approximation. We describe some of this work, due to Majda [25], and Majda and Taylor [28], in \$4.

Time has not permitted us to discuss the recent work of Melrose and Andersson [1], [33], on propagation of singularities near the boundary on the interior of a convex region (the gliding ray problem) or the propagation of singularities of solutions to equations with multiple characteristics, where the 
theorem of Hörmander mentioned above gives an inadequate description, on which progress has been made by Sjöstrand [43], Chazarain [4], Melrose [32], and others. Nor do we discuss the propagation of analytic singularities, for which we refer the reader to [42], [3], and [18].

We will use the following notation for pseudo differential operators and symbol classes. $S_{\rho, \delta}^{m}$ is the symbol class used by Hörmander in [17]. We say $p(x, \xi) \in S_{\rho, \delta}^{m}$ provided

$$
\left|D_{x}^{\beta} D_{\xi}^{\alpha} p(x, \xi)\right|<C_{\alpha \beta}(1+|\xi|)^{m-\rho|\alpha|+\delta|\beta|} .
$$

We say $p(x, \xi) \in S^{m}$ if $p(x, \xi)$ is asymptotic to a sum of symbols homogeneous of degree $m, m-1, m-2$, etc.; $S^{m} \subset S_{1,0}^{m}$. If $\Sigma$ is some symbol class and $p(x, \xi) \in \Sigma$, we say the operator $p(x, D)$ belongs to OP $\Sigma$. Thus we use the operator classes OP $S_{\rho, 0}^{m}$, OP $S^{m}$, etc.

1. Fourier integral operators and propagation of singularities. The basic phenomenon of propagation of singularities can be obtained by analyzing the first order hyperbolic pseudo differential equation

$$
\begin{aligned}
\frac{\partial}{\partial t} u & =i \lambda\left(t, x, D_{x}\right) u+g, \\
u(0) & =f
\end{aligned}
$$

where we suppose $\lambda\left(t, x, D_{x}\right)$ is a smooth family of first order classical pseudo differential operators,

$$
\lambda\left(t, x, D_{x}\right) w=\int \lambda(t, x, \xi) e^{i x \cdot \xi} \hat{w}(\xi) d \xi
$$

with $\lambda(t, x, \xi) \sim \lambda_{1}(t, x, \xi)+\lambda_{0}(t, x, \xi)+\cdots, \lambda_{j}$ being homogeneous of degree $j$ in $\xi$. We assume $\lambda_{1}(t, x, \xi)$ is real valued. Existence and uniqueness of solutions to (1.1), given $g \in H^{s}$ and $u(0)=f \in H^{s}$, follows from simple energy estimates; see for example [46, Chapter IV]. We can construct an approximate solution to (1.1), (1.2) (in case $g=0$ ) as a Fourier integral operator, of the form

$$
v(t, x)=\int a(t, x, \xi) e^{i \varphi(t, x, \xi)} \hat{f}(\xi) d \xi .
$$

Here $a(t, x, \xi)$ is a classical symbol, $a(t, x, \xi) \sim \sum_{j=0}^{\infty} a_{j}(t, x, \xi)$ with $a_{j}$ homogeneous in $\xi$ of degree $-j$. The "phase function" $\varphi$ is real valued and homogeneous of degree 1 in $\xi$, and $\left|\nabla_{x} \varphi\right| \neq 0$. The amplitude $a$ and phase function $\varphi$ are obtained as follows. Applying $\partial / \partial t-i \lambda$ to (1.3) yields

$$
\left(\frac{\partial}{\partial t}-i \lambda\right) v=\int\left(i \varphi_{t} a+a_{t}-i b\right) e^{i \varphi \hat{f}}(\xi) d \xi
$$

where $b(t, x, \xi)$ is defined by

$$
\lambda\left(t, x, D_{x}\right)\left(a e^{i \varphi}\right)=b e^{i \varphi} .
$$

The fundamental asymptotic expansion lemma for pseudo differential operators implies that $b$ is a symbol of classical type, and we have

$$
\left.b(t, x, \xi) \sim \sum_{\alpha>0} \frac{1}{\alpha !} \lambda^{(\alpha)}(t, x, \nabla \varphi) D_{y}^{\alpha}\left(a(t, y, \xi) e^{i \rho\left(x_{y}, \xi\right)}\right)\right|_{y=x}
$$


where $\rho(x, y, \xi)=\varphi(t, y, \xi)-\varphi(t, x, \xi)-(y-x) \cdot \nabla_{x} \varphi(t, x, \xi)$. In particular, the principal symbol of $b$ is $a_{0} \lambda_{1}\left(t, x, \nabla_{x} \varphi\right)$. In order that the right hand side of (1.4) be smooth, we require that the full asymptotic expansion of $i \varphi_{t} a+a_{t}-i b$ vanish. Setting the principal part equal to zero we get the eikonal equation

$$
\varphi_{t}=\lambda_{1}\left(t, x, \nabla_{x} \varphi\right)
$$

This is a first order nonlinear equation, which has a solution for small $|t|$ if $\varphi(0, x, \xi)$ is specified. We set $\varphi(0, x, \xi)=x \cdot \xi$. Setting further terms equal to zero, we get linear differential equations for the $a_{j}(t, x, \xi)$, called the transport equations. For example,

$$
\left(\frac{\partial}{\partial t}-\sum_{j=1}^{n} \frac{\partial \lambda_{1}}{\partial \xi_{j}} \frac{\partial}{\partial x_{j}}\right) a_{0}-\left(i \lambda_{0}+\sum_{|\alpha|=2} \lambda_{1}^{(\alpha)} \varphi_{(\alpha)}\right) a_{0}=0 .
$$

We specify as initial condition that $a_{0}(0, x, \xi)=1$; for $j \geqslant 1$ we set $a_{j}(0, x, \xi)$ $=0$. Thus (1.3) leads to $v(0, x)=f(x)$, by the Fourier inversion formula, while (1.4) implies that $(\partial / \partial t-i \lambda) v$ is smooth. Energy estimates imply that $v$ differs from the exact solution $u$ to $(\partial / \partial t-i \lambda) u=0, u(0)=f$, by a smooth error.

In order to make sure (1.3) is well defined for distributions $f \in \mathcal{E}^{\prime}(\Omega)$, and to justify (1.4), we use an integration by parts procedure similar to the method of defining the Fourier transform of a tempered distribution. Note that $L e^{i \varphi}=i e^{i \varphi}$ if $L=\left|\nabla_{x} \varphi\right|^{-2} \nabla_{x} \varphi \cdot \nabla_{x}$, which is a vector field whose coefficients are homogeneous in $\xi$ of degree -1 . If $M=L^{t}$ is the formal adjoint of $L$, and if $v \in C_{0}^{\infty}$, we have, formally

$$
\begin{aligned}
\left\langle v, \int a e^{i \varphi} \hat{f}(\xi) d \xi\right\rangle & =\iint v(x) a(t, x, \xi) e^{i \varphi \hat{f}}(\xi) d \xi d x \\
& =\iint M^{k}(v a) e^{i \varphi} \hat{f}(\xi) d \xi d x
\end{aligned}
$$

(one need only worry about the integral over $|\xi| \geqslant 1$ ). If $a \in S^{0}$ as above, we see that $M^{k}(v a)$ has order $-k$ in $\xi$. Since for any $f \in \mathcal{E}^{\prime}, \hat{f}(\xi)$ has at most polynomial growth, we see that, for $k$ large enough, the last integral in (1.7) will be absolutely convergent. We can take this formula to define (1.3).

For any fixed $t$, the wave front set of $v(t, x)=A(t) f$ can be analyzed as follows. To say $\left(x_{0}, \xi_{0}\right) \notin \mathrm{WF}(A(t) f)$ is equivalent to saying that for some $\chi \in C_{0}^{\infty}, \chi(x)=1$ near $x_{0},\left\langle\chi(x) e^{-i x \cdot \theta}, A(t) f\right\rangle$ is rapidly decreasing as $\theta \rightarrow \infty$ on some conic neighborhood $\Gamma$ of $\xi_{0}$. Thus we consider

$$
\begin{gathered}
\left\langle\chi(x) e^{-i x \cdot \theta}, A(t) f\right\rangle \\
=\iiint f(y) \chi(x) a(t, x, \xi) e^{i \varphi(t, x, \xi)-i y \cdot \xi-i x \cdot \theta} d y d x d \xi
\end{gathered}
$$

the integral with respect to $y$ being taken in the distribution sense, and the $\xi$ integral being regarded as an oscillatory integral, like (1.3).

Before we proceed with the analysis of (1.8), let us make some preliminary observations that will simplify the analysis. Suppose $\operatorname{WF}(f)$ is contained in a small conic neighborhood of $\left(y_{0}, \eta_{0}\right)$. We may as well suppose that $f(y)$ is 
supported near $y_{0}$. Also, since $A(t) f$ defined by (1.3) would only be altered by a smooth function, we may as well suppose that $a(t, x, \xi)$ is supported for $\xi$ in a small conic neighborhood of $\eta_{0}$, and that $a(t, x, \xi)=0$ for $|\xi| \leqslant 1$.

With these hypotheses, we can show that (1.8) is rapidly decreasing as $\theta \rightarrow \infty$ in a cone $\Gamma$ with the property that, for $\theta \in \Gamma$, the function $\Phi=$ $\varphi(t, x, \xi)-y \cdot \xi-x \cdot \theta$ has no critical point as a function of $x$ and $\xi$, i.e., assuming that, for $\theta \in \Gamma,\left|\nabla_{x} \varphi-\theta\right|+|\xi|\left|\nabla_{\xi} \varphi-y\right|$ is bounded away from 0 (on $|\xi|,|\theta| \geqslant 1$ ). Indeed, let

$$
\begin{gathered}
L=\left[\left|\nabla_{x} \varphi-\theta\right|^{2}+(|\xi|+|\theta|)^{2}\left|\nabla_{\xi} \varphi-y\right|^{2}\right]^{-1} \\
\cdot\left[\left(\nabla_{x} \varphi-\theta\right) \cdot \nabla_{x}+(|\xi|+|\theta|)^{2}\left(\nabla_{\xi} \varphi-y\right) \cdot \nabla_{\xi}\right],
\end{gathered}
$$

which yields $L e^{i \Phi}=i e^{i \Phi}$. By hypothesis, the coefficients of $L$ are smooth for $\theta \in \Gamma,(t, x, \xi) \in \operatorname{supp} a$. It follows that

$$
\left\langle\chi(x) e^{-i \cdot \theta}, A(t) f\right\rangle=\iiint f(y)\left(L^{t}\right)^{k}(\chi(x) a(t, x, \xi)) e^{i \Phi} d y d x d \xi .
$$

Taking $k$ large, one can show without difficulty that this is rapidly decreasing as $\theta \rightarrow \infty$ in $\Gamma$. Since any $f \in \mathcal{E}^{\prime}$ may be decomposed into a finite sum of $f_{j} \in \mathcal{E}^{\prime}$ with small wave front sets, this argument establishes the following.

Proposition 1.1. If $A(t) f$ is given by (1.3), then

$$
\begin{aligned}
\mathrm{WF}(A(t) f) \subset\{(x, \theta): & \left(\nabla_{\xi} \varphi, \xi\right) \in \mathrm{WF}(f) \text { for some } \\
& \left.(x, \xi) \in \text { conic supp } a \text { and } \nabla_{x} \varphi(t, x, \xi)=\theta\right\} .
\end{aligned}
$$

Thus $\mathrm{WF}(f)$ and $\mathrm{WF}(A(t) f)$ are related by the canonical relation

$$
\left(\nabla_{\xi} \varphi, \xi\right) \mapsto\left(x, \nabla_{x} \varphi\right) .
$$

Given the eikonal equation (1.5) for $\varphi$, one can show that this transformation is precisely the flow generated by $H_{\lambda_{1}}$. Rather than giving the details of this here, we will give a different argument relating $\mathrm{WF}(u(t))$ to $\mathrm{WF}(f)$, based on Egorov's theorem.

Egorov's theorem analyzes the operator $A(t) P B(t)$, where $A(t)$ is the solution operator to $(\partial / \partial t) u=i \lambda u$ defined above, and $B(t)$ solves the backwards equation, i.e., if $(\partial / \partial t) u=i \lambda u$ and $u(t)=f, u(0)=B(t) f . P=$ $p(x, D)$ is a scalar pseudo differential operator with symbol $p(x, \xi) \in S_{1,0}^{m}$, i.e., $\left|D_{x}^{\beta} D_{\xi}^{\alpha} p(x, \xi)\right| \leqslant c(1+|\xi|)^{m-|\alpha|}$. In that case, we have

EGOROV'S THEOREM. $Q(t)=A(t) P B(t)$ is a pseudo differential operator, on a compact manifold $\Omega$, whose principal symbol $\left(\bmod S_{1,0}^{m-1}\right)$ is equal to

$$
q(t, x, \xi)=p(C(t)(x, \xi))
$$

where $C(t)$ is the flow generated by the Hamiltonian vector field $H_{\lambda_{1}}$.

Proof. We briefly sketch a proof. We construct an approximate solution $\tilde{Q}$ to the equation $Q^{\prime}(t)=d / d t(A(t) P B(t))$, i.e.

$$
\tilde{Q}^{\prime}(t)=i \lambda \tilde{Q}(t)-i \tilde{Q}(t) \lambda \bmod \text { OP } S^{-\infty}
$$


with $\tilde{Q}(0)=P$, such that $\tilde{Q}(t)$ is a pseudo differential operator satisfying (1.9), and then we show that $\tilde{Q}(t)$ differs from $Q(t)$ by a smoothing operator.

We specify the symbol $\tilde{q}(t, x, \xi)$ of $\tilde{Q}(t)$ so that $(\partial / \partial t) \tilde{q}-i \sigma_{[\lambda, \tilde{Q}]}$ is of order $-\infty$, where

$$
\sigma[\lambda, \tilde{Q}] \sim \sum_{|\alpha|>1} \frac{i^{|\alpha|}}{\alpha !}\left\{\left(D_{\xi}^{\alpha} \lambda\right)\left(D_{x}^{\alpha} \tilde{q}\right)-\left(D_{\xi}^{\alpha} \tilde{q}\right)\left(D_{x}^{\alpha} \lambda\right)\right\}
$$

We arrange this by setting $\tilde{q} \sim \Sigma_{\nu \geqslant 0} \tilde{q}_{\nu}(t, x, \xi)$ with $\tilde{q}_{\nu} \in S_{1,0}^{m-\nu} . \tilde{q}_{0}(t, x, \xi)$ satisfies the transport equation $\left(\partial / \partial t-H_{\lambda_{1}}\right) \tilde{q}_{0}=0$, with initial condition $\tilde{q}_{0}(0, x, \xi)=p(x, \xi)$. The $\tilde{q}_{\nu}$ for $\nu \geqslant 1$ satisfy other transport equations, and $\tilde{q}_{v}(0, x, \xi)=0$. Then (1.9) is satisfied for $\tilde{q}$.

Showing that $Q(t)-\tilde{Q}(t)$ is smoothing is equivalent to showing $A(t) P-$ $\tilde{Q}(t) A(t)$ is smoothing. But if $u(t)=A(t) P f$ and $v(t)=\tilde{Q}(t) A(t) f$, then $u(0)-v(0)=0$ while $(\partial / \partial t-i \lambda)(u-v)$ is smooth. Energy estimates for the hyperbolic operator $\partial / \partial t-i \lambda$ imply that $u-v$ is smooth, given any distribution $f$. Thus $Q(t)-\tilde{Q}(t)$ is smoothing.

For further details of this proof, see Chapter I of [47].

Given Egorov's theorem, we will establish the following result on propagation of singularities.

THEOREM 1.2. If the solution $u$ to $(\partial / \partial t-i \lambda) u=0, u(0)=f$ is $u(t)=$ $A(t) f$, then

$$
\mathrm{WF}(u(t))=C(t) \mathrm{WF}(u(0))
$$

where $C(t)$ is the one parameter flow generated by $H_{\lambda_{1}}$.

In order to prove this result, we use a different characterization of $\operatorname{WF}(f)$, also due to Hörmander, which uses pseudo differential operators. If $p(x, \xi) \sim$ $\sum_{j=0}^{\infty} p_{j}(x, \xi)$ with $p_{j}(x, \xi)$ homogeneous of degree $-j$ in $\xi$, we say $p \in S^{0}$, and $p(x, D) \in \mathrm{OP} S^{0}$. Char $p=\left\{(x, \xi): p_{0}(x, \xi)=0\right\}$. Then

$$
\mathrm{WF}(f)=\bigcap\left\{\operatorname{char} p: p(x, D) f \in C^{\infty}, p \in S^{0}\right\} .
$$

For the equivalence of these two definitions of WF( $f)$, see [7] or [15].

PROOF OF THEOREM 1.2. Let $p_{j}(x, D)$ be a family of operators in OP $S^{0}$, each of whose symbols vanishes in a neighborhood of WF $(f)$, but such that $\mathrm{WF}(f)=\cap_{j}$ char $p_{j}$. Clearly $p_{j}(x, D) f \in C^{\infty}$ for each $j$. If $g=A(t) f$, this implies that $A(t) p_{j}(x, D) B(t) g \in C^{\infty}$. But by Egorov's theorem this is a pseudo differential operator, say $q_{j}(t, x, D)$, and char $q_{j}(t, x, D)=C(t)$ char $p_{j}$. It follows that

$$
\mathrm{WF}(A(t) f) \subset \bigcap_{j} C(t) \operatorname{char} p_{j}=C(t) \mathrm{WF}(f) .
$$

Reversibility of the hyperbolic equation $(\partial / \partial t-i \lambda) u=0$ yields to reverse inclusion, so $\mathrm{WF}(A(t) f)=C(t) \mathrm{WF}(f)$, as asserted.

Note that the computation of $u(t)$ via the geometrical optics construction (1.3) yields Theorem 1.2 for small $t$. Similarly, it yields $\operatorname{WF}(u(t))=$ $C(t, s) \mathrm{WF}(u(s))$, where $C(t, s)$ is the flow generated by the time dependent vector field $H_{\lambda_{1}}$, from time $s$ to time $t$, provided $s$ and $t$ are sufficiently close. A connectedness and compactness argument then yields Theorem 1.2. In 
order to prove Theorem 1.2 in a fell swoop this way one would need a global construction of a parametrix, such as given in [8]. One way to obtain a global parametrix is as a product of solution operators with short time steps. The analysis of products of Fourier integral operators and the global construction of parametrices form some of the deepest parts of the theory of Fourier integral operators, and we refer the reader to Hörmander [15], Duistermaat and Hörmander [8], and Duistermaat [7] for discussion of these topics.

Theorem 1.2 is not really equivalent to Hörmander's theorem, but we can obtain that result by a simple trick. Thus if $P u=f, P$ has order $m$, with real principal part, choose an elliptic operator $E$ of order $1-m$ and let $\lambda=E P$, so you get $\lambda u=E f=g$. Now introduce an extra variable, $t$, and let $v(t, x)=$ $u(x)$. Then $(\partial / \partial t) v=0$, so you have

$$
\begin{aligned}
\left(\frac{\partial}{\partial t}-i \lambda\right) v & =-i g, \\
v(0) & =u .
\end{aligned}
$$

From this it is not hard to deduce, via Theorem 1.2, that $\mathrm{WF}(u) \backslash \mathrm{WF}(f)$ is invariant under the Hamiltonian flow generated by $H_{\lambda_{1}}$ on $T^{*}(\Omega) \backslash W F(f)$.

If $P$ is not a scalar but a $k \times k$ matrix of operators, one can analyze the singularities of solutions to $P u=f$ by multiplying by a convenient operator $E$, of order $1-m$, so that the principal symbol $q_{1}$ of $E P$ is scalar. For example, one could take the principal symbol of $E$ to be $|\xi|^{1-k m}$ times the cofactor matrix of $\boldsymbol{P}_{m}$, so $q_{1}=|\xi|^{1-k m}$ det $\boldsymbol{P}_{m}$. However, for many systems one encounters in practice, it is best not to take the determinant of the principal symbol. To take a trivial example, suppose $P$ is a $2 \times 2$ system whose principal symbol $P_{m}(x, \xi)=p_{m}(x, \xi) I$, where $p_{m}(x, \xi)$ is scalar, real valued. If $P u \in C^{\infty}, \operatorname{WF}(u) \subset\left\{(x, \xi): p_{m}(x, \xi)=0\right\}$. The above method implies that WF $(u)$ is invariant under the flow on $T^{*}(\Omega)$ generated by $H_{a}$, with $q_{1}(x, \xi)=$ $|\xi|^{1-2 m_{p}} p_{m}(x, \xi)^{2}$. But clearly all characteristics of $q_{1}$ are double, so $H_{q_{1}}=0$ on char $P$, and hence the flow $C(t)$ is the identity on char $P$, os we get no information about propagation of singularities by taking the determinant. However, the obvious result of interest is that $\operatorname{WF}(u)$ is invariant under the flow generated by $H_{\tilde{q}_{1}}$ with $\tilde{q}_{1}=|\xi|^{1-m_{p}} p_{m}$.

A more important example of this phenomenon is given by the equations of linear elasticity for an isotropic medium:

$$
L u=\frac{\partial^{2}}{\partial t^{2}} u-(\lambda+\mu) \operatorname{grad} \operatorname{div} u-\mu \Delta u=0
$$

where $u=u(t, x)$ is a 3-vector field on $\mathbf{R} \times \mathbf{R}^{3}$. The quantities $\lambda$ and $\mu$, called the Lame constants, are assumed positive. The principal symbol of $L$ is $L_{2}(t, x, \tau, \xi)=-\tau^{2} I+(\lambda+\mu)|\xi|^{2} P_{\xi}+\mu|\xi|^{2}$ where $P_{\xi}$ is the orthogonal projection of $\mathbf{R}^{3}$ onto the space spanned by $\xi$. For each $(\tau, \xi) \neq 0$, this is a symmetric matrix, with a simple eigenvalue $(\lambda+2 \mu)|\xi|^{2}-\tau^{2}$ and a double eigenvalue $\mu|\xi|^{2}-\tau^{2}$. The analysis of propagation of singularities of solutions to these equations of linear elasticity is a special case of the following result, which can be proved in the manner indicated above.

COROLlary 1.3. Let $P_{m}(x, \xi)$ be a $k \times k$ selfadjoint matrix, and suppose 0 is 
an eigenvalue of multiplicity exactly $m_{j}$ on each connected component $\Gamma_{j}$ of char $P=\left\{(x, \xi)\right.$ : det $\left.P_{m}(x, \xi)=0\right\}$. Furthermore, suppose that there is an eigenvalue $\mu_{j}(x, \xi)$, of multiplicity exactly $m_{j}$ on some conic neighborhood of $\Gamma_{j}$, with $\mu_{j}(x, \xi)=0$ on $\Gamma_{j}, \mu_{j}$ smooth. If $P u=f$, then $\operatorname{WF}(u) \subset \cup_{j} \Gamma_{j} \cup \operatorname{WF}(f)$, and $\left(\mathrm{WF}(u) \cap \Gamma_{j}\right) \backslash \mathrm{WF}(f)$ is invariant under the flow (on $\left.\Gamma_{j} \backslash \mathrm{WF}(f)\right)$ generated by $H_{|\xi|}{ }^{1-m_{\mu}}$.

For further details on propagation of singularities from the point of view espoused in this section, we refer the reader to Chapter I of [47].

2. Reflection of singularities. In this section we shall examine reflection of singularities of solutions of first order equations of the form

$$
\frac{\partial}{\partial y} u=G u
$$

in a region $\Omega$ with boundary $\partial \Omega$ given by $y=0$; say $\Omega=\mathbf{R}^{+} \times \partial \Omega$. Here $G=G(y)=G\left(y, x, D_{x}\right)$ is a smooth one parameter family of pseudo differential operators of order one on $\partial \Omega, G(y) \in \mathrm{OP} S^{1} ; u$ takes values in a vector space, $\mathbf{C}^{k}$, and $G$ is a $k \times k$ matrix of operators, with principal symbol $G_{1}(y, x, \xi)$, homogeneous of degree one in $\xi$. We suppose the characteristics of $\partial / \partial t-G$ are simple. On the boundary $y=0$, a boundary condition is prescribed:

$$
B u(0)=f
$$

where $B \in O P S^{0}$ is a pseudo differential operator of order zero.

There is no loss of generality in dealing with first order systems, since higher order equations can be reduced to first order systems by a standard argument (see, for example, Chapters IV and V of [46]).

We suppose that $f$ has wave front set in a small conic neighborhood of $\left(x_{0}, \xi_{0}\right) \in T^{*}(\partial \Omega)$, which is no real restriction. Suppose that $j$ null bicharacteristic strips $\gamma_{1}, \ldots, \gamma_{j}$ pass over $\left(x_{0}, \xi_{0}\right) \in T^{*}(\partial \Omega)$. We are treating the nongrazing case in this section, so we suppose the $\gamma_{\nu}$ all intersect $\partial \Omega$ transversally. It's not too hard to see that this hypothesis implies that the principal symbol of $G(y)$ is similar to a matrix of the form

$$
\left[\begin{array}{lllll}
i \lambda_{1} & & & & \\
& \ddots & & & \\
& & i \lambda_{j} & & \\
& & & A_{-} & \\
& & & & A_{+}
\end{array}\right]=\tilde{G}_{1}
$$

near $\left(x_{0}, \xi_{0}\right)$, where $\lambda_{\nu}(y, x, \xi)$ are real valued (scalar), the spectrum of $A_{-}(y, x, \xi)$ has negative real part, and the spectrum of $A_{+}(y, x, \xi)$ has positive real part. This similarity can be effected, near $\left(x_{0}, \xi_{0}\right)$, by an invertible matrix function $U(y, x, \xi) ; \tilde{G}_{1}=U G_{1} U^{-1}$ near $\left(x_{0}, \xi_{0}\right)$.

If $\psi(x, \xi)$ is a zero order symbol supported in a small conic neighborhood $U_{0}$ of $\left(x_{0}, \xi_{0}\right)$ and equal to 1 on a smaller conic neighborhood, let $v=$ $U(y, x, D) \psi(x, D) u$. Then $v$ solves the system 


$$
\frac{\partial}{\partial y} v=\tilde{G} v+F
$$

with boundary condition

$$
U B U^{-1} v(0)=\tilde{f} .
$$

Here $\tilde{G}=U G U^{-1}+U_{y} U^{-1} \in \mathrm{OP} S^{1}$ has principal symbol $\tilde{G}_{1}(y, x, \xi) . F=$ $U[\psi, G] u$ and $\tilde{f}=U[B, \psi] u(0)+U f$, plus smooth functions. With (2.4) we have decoupled the equation (2.1), at least in the principal part. There is coupling in the zero order terms. It is convenient to completely decouple (2.4), modulo a smoothing operator. We briefly describe how this can be accomplished.

We consider a slightly more general problem. Let $v$ solve the system

$$
\frac{\partial}{\partial y} v=\left(\begin{array}{ll}
F & E
\end{array}\right) v+A v
$$

where $G=\left({ }_{E}\right)$ has symbol homogeneous of order 1 and $A$ has order zero. The assumption we shall make on the symbols $F(y, x, \xi)$ and $E(y, x, \xi)$ is that these two matrices have disjoint sets of eigenvalues, for each $(y, x, \xi)$. First we decouple terms of order zero by a substitution $w^{(1)}=\left(1+K_{1}\right) v$, with $K_{1} \in \mathrm{OP} S^{-1}$ chosen appropriately. This yields.

$$
\frac{\partial}{\partial y} w^{(1)}=G w^{(1)}+\left(K_{1} G-G K_{1}+A\right) w^{(1)}+\cdots .
$$

If we specify

$$
K_{1}=\left(\begin{array}{cc}
0 & K_{12} \\
K_{21} & 0
\end{array}\right)
$$

making the off-diagonal term of $K_{1} G-G K_{1}+A$ vanish

$$
\left(\operatorname{say} A=\left(\begin{array}{ll}
A_{11} & A_{12} \\
A_{21} & A_{22}
\end{array}\right)\right)
$$

is equivalent to having, on the symbol level

$$
\begin{aligned}
& K_{12} F-E K_{12}=-A_{12}, \\
& K_{21} E-F K_{21}=-A_{21} .
\end{aligned}
$$

Since the spectra of $E$ and $F$ are assumed disjoint, it is a simple linear algebra exercise to obtain unique solutions $K_{12}$ and $K_{21}$ for (2.7). This decouples the zero order part of (2.6). One can continue in this fashion, setting $w^{(2)}=(1+$ $\left.K_{2}\right) w^{(1)}$, with $K_{2} \in$ OP $S^{-2}$, etc. Finally, let $w=(1+K) v$ with

$$
1+K \sim \cdots\left(1+K_{2}\right)\left(1+K_{1}\right)
$$

to obtain an equation for $w$ which is completely decoupled, modulo a smoothing operator.

Applying this decoupling procedure inductively, we can write $w=(1+$ $K) v$ with $K \in \mathrm{OP} S^{-1}$ such that 


$$
w=\left(\begin{array}{c}
w_{1} \\
\vdots \\
w_{j} \\
w_{+} \\
w_{-}
\end{array}\right),
$$

where, modulo unimportant inhomogeneous terms, $w_{p}$ solve the equations

$$
\frac{\partial}{\partial y} w_{\nu}=i \mu_{\nu}\left(y, x, D_{x}\right) w_{\nu}
$$

and $w_{+}$and $w_{-}$solve, respectively

$$
\begin{aligned}
& \frac{\partial}{\partial y} w_{+}=a_{-}(y, x, D) w_{+}, \\
& \frac{\partial}{\partial y} w_{-}=a_{+}(y, x, D) w_{-}
\end{aligned}
$$

and furthermore the principal symbol of $\mu_{\nu}$ is $\lambda_{\nu}$, the principal symbol of $a_{-}\left(y, x, D_{x}\right)$ is $A_{-}$, and the principal symbol of $a_{+}(y, x, D)$ is $A_{+}$. The boundary condition (2.2) becomes essentially

$$
B U^{-1}(1+K)^{-1} w(0)=f .
$$

The reflection of singularities phenomenon we consider is described simply as follows. Suppose we know that $u$ is smooth in a conic neighborhood of the rays $\gamma_{1}, \ldots, \gamma_{l}(0 \leqslant l \leqslant j)$ passing over $\left(x_{0}, \xi_{0}\right) \in T^{*}(\partial \Omega)$, where $\gamma_{v}$ is a null bicharacteristic strip associated to $\partial / \partial y-i \lambda_{\nu}$. Note that this is equivalent to the smoothness (up to the boundary $y=0$ ) of $w_{1}, \ldots, w_{l}$. More generally, suppose we know the nature of the singularities of $u$ near $\gamma_{1}, \ldots, \gamma_{l}$, i.e., suppose we know $w_{1}, \ldots, w_{l}, \bmod C^{\infty}$. We want to construct a parametrix for $u(y)$, which in particular will tell us the nature of the singularities of $w_{l+1}, \ldots, w_{j}$, and also the boundary regularity of $w_{+}$, hence the complete nature of the singularities of $u$. (Note that since $w_{+}$and $w_{-}$solve elliptic evolutions, forward and backward, respectively, they are automatically $C^{\infty}$ inside $(0, Y) \times \partial \Omega$ and $w_{-}$is smooth up to the boundary $y=0$.) The following result is a consequence of the conversion of (2.1), (2.2) into (2.8)-(2.11).

THEOREM 2.1. Suppose that, given the values of $w_{1}(0), \ldots, w_{l}(0)$ and of $w_{-}(0)$, the system $(2.11)$ is an elliptic system for $w_{l+1}(0), \ldots, w_{j}(0), w_{+}(0)$. Then smoothness of $u$ along $\gamma_{1}, \ldots, \gamma_{1}$ implies smoothness of $u$ along $\gamma_{t+1}, \ldots, \gamma_{j}$, provided $\left(x_{0}, \xi_{0}\right) \notin \mathrm{WF}(f)$. Furthermore the parametrix for $u$ can be constructed by solving (2.11) for $w_{l+1}(0), \ldots, w_{j}(0), w_{+}(0)$, then solving (2.8) for $w_{l+1}(y), \ldots, w_{j}(y)$ and (2.9) for $w_{+}(y)$, and then writing $u=U^{-1}(1+$ $K)^{-1} w$.

Examples where this theorem works include the Dirichlet and Neumann problems for the wave equation $\left(\partial^{2} / \partial t^{2}-\Delta\right) u=0$, reduced to a first order system, assuming that tangential bicharacteristics don't pass over $\left(x_{0}, \xi_{0}\right)$. In this case, over each $\left(x_{0}, \xi_{0}\right) \in T^{*}(\partial \Omega)$ passes either two rays, which are 
related by angle of incidence equals angle of reflection, or one ray (grazing), or no rays. The transmission problem is also included in Theorem 2.1. If $S$ is a surface in $R^{3}$, dividing $R^{3}$ into two regions, $\Omega_{1}$ and $\Omega_{2}$, we suppose

$$
\begin{array}{ll}
\left(\partial^{2} / \partial t^{2}-C_{1}^{2} \Delta\right) u=0 & \text { in } \mathbf{R} \times \Omega_{1}, \\
\left(\partial^{2} / \partial t^{2}-C_{2}^{2} \Delta\right) v=0 & \text { in } \mathbf{R} \times \Omega_{2}
\end{array}
$$

with $\left.u\right|_{s}=\left.v\right|_{s}$ and $\left.\nabla u\right|_{s}=\left.\nabla v\right|_{s}$. Suppose $C_{1} \neq C_{2}$ on $K$. In this case, over each point in $T^{*}(R \times S) \backslash 0$ pass either 4 rays (the "hyperbolic" region), 3 rays (1 grazing), 2 rays (the "mixed" region), 1 ray (grazing), or 0 rays (the "elliptic" region). All nongrazing cases are treated by Theorem 2.1. The directions over which 1 grazing ray and 2 nongrazing rays pass represent the critical directions bordering total internal reflection of rays hitting $S$ from the slow sound speed region. In [48] a construction is given showing how a laser beam aimed at $S$ and hitting it at such a critical angle can shoot off a two-dimensional sheet of singularities.

The Dirichlet problem is an example of a well posed boundary value problem in the sense of Kreiss [20] and Sakamoto [41]. As shown in [48], for all such boundary value problems, analysis of reflection of singularities in the nongrazing case follows from Theorem 2.1. The Neumann boundary value problem, and also the boundary value problems for electromagnetic waves on the exterior of a perfectly conducting obstacle, do not satisfy the KreissSakamoto condition, but this condition fails only at the grazing directions. This makes the parametrix for the diffraction problem a little different from that for the Dirichlet problem, but doesn't affect the nongrazing analysis.

One important problem for which the Kreiss-Sakamoto condition fails in nongrazing directions is the following problem from linear elasticity:

$$
L u=\frac{\partial^{2}}{\partial t^{2}} u-(\lambda+\mu) \operatorname{grad} \operatorname{div} u-\mu \Delta u=0
$$

already discussed in $\$ 1$ (see (1.11)) with the boundary condition

$$
\sum_{i} n_{i} \sigma_{i j}=0 \text { on } \partial K
$$

where $\sigma_{i j}$ is the stress tensor: $\sigma_{i j}=\lambda(\operatorname{div} u) \delta_{i j}+\mu\left(\partial u_{i} / \partial x_{j}+\partial u_{j} / \partial x_{i}\right)$. Here $n_{i}$ represents the components of the unit normal to $\partial K$. As mentioned in $\$ 1$, (2.12) has two sound speeds. Consequently, as for the transmission problem, $T^{*}(\mathbf{R} \times \partial K)$ is divided into 3 regions. It turns out that the Kreiss-Sakamoto condition fails on a conic hypersurface in the "elliptic" region of $T^{*}(\mathrm{R} \times \partial K)$ (over which no rays pass). Theorem 2.1 does not apply to this boundary value problem. Indeed, when (2.12) is converted to a first order system, the boundary pseudo differential operator one obtains to analyze (2.13) is not elliptic. It turns out that, upon multiplication by an elliptic scalar, the operator one gets has real determinant, with simple characteristics. Consequently a unique solution $\left(\bmod C^{\infty}\right)$ to the associated boundary condition can be found, and its singularities analyzed by Hörmander's theorem on propagation of singularities. Such singularities propagate at a speed a little less than the slower sound speed of (2.12). The associated solutions to (2.12) have singularities which run along the boundary at such a 
speed. These are called Rayleigh waves, and were first studied for flat boundaries in [40]. For the details of the analysis in the presence of curved boundaries, see [52]. A generalization of the analysis leading to Theorem 2.1 yields the following result, which contains the analysis of Rayleigh waves.

THEOREM 2.2. One can construct a solution $\left(\bmod C^{\infty}\right)$ to (2.1), (2.2), given $f \in \mathcal{E}^{\prime}(\partial \Omega)$, with the property that $u$ is smooth along the rays $\gamma_{1}, \ldots, \gamma_{l}$, provided that, for specified $w_{1}(0), \ldots, w_{l}(0), w_{-}(0) \in C^{\infty}(\partial \Omega)$, we can solve the system

$$
B U(0)^{-1}(1+K)^{-1} w(0)=f\left(\bmod C^{\infty}\right)
$$

for $w_{l+1}(0), \ldots, w_{j}(0), w_{+}(0)$. If we can deduce that $\mathrm{WF} w_{\nu}(0) \subset \Gamma_{\nu}, \mathrm{WF} w_{+}(0)$ $\subset \Gamma_{+}$, where $\Gamma_{\nu}(\nu=l+1, \ldots, j)$ and $\Gamma_{+}$are closed conic subsets of $T^{*}(\partial \Omega)$ obtained from WF $(f)$ by some process, it follows that WF(u) is smooth except along those rays passing over $\cup_{\nu} \Gamma_{\nu}$. Furthermore, $w_{+}(y)$ is smooth up to the boundary $y=0$ except at points $x \in \partial \Omega$ such that $(x, \xi) \in \Gamma_{+}$for some $\xi$.

We close by remarking that another approach to the analysis of reflection of singularities, given the results of Lax and Nirenberg [36] for the Dirichlet boundary condition, is to construct a pseudo differential operator $Q$ on $\partial \Omega$ such that if $P u=0,\left.u\right|_{\partial \Omega}=f$, then $\left.B u\right|_{\partial \Omega}=Q f$. Then the analysis of reflection of singularities is reduced to the analysis of $Q$. This approach is taken in Majda and Osher [26]. For further details on the approach discussed in this section, see [48].

3. Grazing rays and diffraction. In this section we treat the diffraction problem, the study of propagation of singularities along rays which hit $\partial \Omega$ tangentially. We assume that such rays remain inside $\Omega$, and have exactly second order contact with $\partial \Omega$. Such rays are called grazing rays. If $\Omega=\mathbf{R} \times$ $\left(\mathbf{R}^{n} \backslash K\right)$ where $K$ is a smooth strictly convex obstacle in $\mathbf{R}^{n}$, then straight lines hitting $\partial \Omega$ tangentially, travelling at speed 1 , give rise to grazing rays for the wave operator $\square=\partial^{2} / \partial t^{2}-\Delta$. Since all the analytical difficulties occur in this case, we will restrict our attention to the wave equation

$$
\left(\frac{\partial^{2}}{\partial t^{2}}-\Delta\right) u=0
$$

on the exterior of a smooth convex obstacle $K \subset \mathbf{R}^{n}$. We will consider either Dirichlet boundary conditions

$$
\left.u\right|_{\partial K}=f
$$

or Neumann conditions

$$
\left.\frac{\partial}{\partial \nu} u\right|_{\partial K}=g
$$

and make brief comments on more general boundary conditions. Here we assume $f, g \in \mathcal{E}^{\prime}(\mathrm{R} \times \partial K)=\mathcal{E}^{\prime}(\partial \Omega)$ and take the unique solution $u$ to (3.1), (3.2) or (3.1), (3.3) which vanishes for $t \ll 0$.

If $\mathrm{WF}(f)$ is disjoint from the set of directions in $T^{*}(\partial \Omega)$ over which grazing rays pass, the construction of $\$ 2$ will work. Since we can write any $f$ as a finite sum of distributions with small wave front sets, we may suppose WF( $f)$ is 
contained in a small conic neighborhood of a point $\left(x_{0}, \zeta_{0}\right) \in T^{*}(\partial \Omega)$ over which a grazing ray passes. We want to construct a solution, $\bmod C^{\infty}$, to (3.1), (3.2) (or (3.1), (3.3)), equal to 0 for $t \ll 0$, which is only singular in a small conic neighborhood of this ray.

To motivate the ansatz we will use, let's consider why the usual geometrical optics ansatz, using operators of the form (1.3), which as we saw in $\$ 2$ works for nongrazing reflection of singularities, fails in the grazing ray case. The reason is that the phase function, which would be real valued in the "hyperbolic" region but complex valued in the "elliptic" region, would fail to be smooth. This corresponds to the fact that the canonical flow of points in $T^{*}(\partial \Omega)$ along rays in $T^{*}(\Omega)$ fails to be smooth at the grazing directions. What is needed is some smooth decomposition of the rays near grazing, and this is provided by a "caustic decomposition" of the rays having caustics on convex surfaces $S_{\alpha}$ tending toward $S_{0}=\partial \Omega$. The grazing rays have as their caustic surface precisely $\partial \Omega$. The construction of solutions with a given convex caustic has been carried out by Ludwig [23] and such constructions involve the Airy function. See also Duistermaat [7].

Recall that the Airy function, defined by

$$
\operatorname{Ai}(s)=\frac{1}{\pi} \int_{0}^{\infty} \cos \left(\frac{1}{3} t^{3}+s t\right) d t
$$

solves the Airy equation

$$
\operatorname{Ai}^{\prime \prime}(s)-s \mathrm{Ai}(s)=0 .
$$

The asymptotic behavior of $\mathrm{Ai}(s)$ is well known (see Erdélyi [10]). $\mathrm{Ai}(s)$ is equal to the sum of two oscillatory terms for $s<0$ and exponentially decreasing for $s>0$. Furthermore, we have the following asymptotic expansion, valid for $-\pi<\arg z<\pi$ :

$$
\operatorname{Ai}(z)=\Phi(z) e^{-(2 / 3) z^{3 / 2}}
$$

with

$$
\Phi(z) \sim z^{-1 / 4}\left(\frac{1}{2 \sqrt{\pi}}+a_{1} z^{-3 / 2}+\cdots\right) \text { as } z \rightarrow \infty .
$$

We will construct an approximate solution to (3.1), (3.2) as a superposition of functions of the form (with $\zeta=(\xi, \eta)$ )

$$
\begin{aligned}
& {\left[g(t, x, \zeta) A\left(|\xi|^{-1 / 3} \rho(t, x, \zeta)\right)\right.} \\
& \left.\quad+i h(t, x, \zeta)|\xi|^{-1 / 3} A^{\prime}\left(|\xi|^{-1 / 3} \rho(t, x, \zeta)\right)\right] e^{i \theta(t, x, \zeta)}
\end{aligned}
$$

where $A(s)=\operatorname{Ai}\left(-e^{(2 / 3) \pi i} s\right)$, also a solution to Airy's equation. From (3.4) we see that $A(s)$ for $s \rightarrow+\infty$ is purely oscillatory, while $A(s)$ has exponential increase as $s \rightarrow-\infty$. In fact, we have

$$
\begin{aligned}
& A(s)=\Phi\left(e^{-\pi i / 3} s\right) e^{-(2 / 3) i s^{3 / 2}}, \quad s>0, \\
& A(s)=\Phi\left(-e^{2 / 3 \pi i}\right) e^{2 / 3(-s)^{3 / 2}}, \quad s<0 .
\end{aligned}
$$

The function $\rho$ will be positive outside the caustic and negative inside. 
With these considerations in mind, we now write down the parametrix for solutions to (3.1), on $\mathbf{R} \times\left(\mathbf{R}^{n} \backslash K\right)$.

$$
\begin{aligned}
u(t, x)=\iint[g & \frac{A\left(|\xi|^{-1 / 3} \rho\right)}{A\left(-|\xi|^{-1 / 3} \eta\right)} \\
& \left.+i h|\xi|^{-1 / 3} \frac{A^{\prime}\left(|\xi|^{-1 / 3} \rho\right)}{A\left(-|\xi|^{-1 / 3} \eta\right)}\right] e^{i \theta} \hat{F}(\xi, \eta) d \xi d \eta
\end{aligned}
$$

where $\rho=\rho(t, x, \xi, \eta)$, and $\theta=\theta(t, x, \xi, \eta)$ will be our phase functions and $g=g(t, x, \xi, \eta)$ and $h=h(t, x, \xi, \eta)$ will be our amplitudes. $\rho$ and $\theta$ will be real valued and homogeneous of degree 1 in $(\xi, \eta)$, and we will have $g \sim \sum_{j \geqslant 0} g_{j}, h \sim \Sigma_{j \geqslant 0} h_{j}$ with $g_{j}, h_{j}$ homogeneous of degree $-j$ in $(\xi, \eta)$. The distribution $F$ is an unknown, which will be determined by the boundary condition.

To guarantee that the function $u$ given by (3.5) solves the wave equation, up to a smooth error, we apply the wave operator to (3.4) and separate out terms of like order. We use the Airy equation for $A(s)$ to express the integrand in terms of $A\left(|\xi|^{-1 / 3} \rho\right)$ and $A^{\prime}\left(|\xi|^{-1 / 3} \rho\right)$. We regard $A^{\prime}\left(|\xi|^{-1 / 3} \rho\right)$ as having order $\frac{1}{3}$ greater than $A\left(|\xi|^{-1 / 3} \rho\right)$, as suggested by differentiating (3.4). Setting the top order term equal to zero yields the eikonal equations for $\rho$ and $\boldsymbol{\theta}:$

$$
\begin{gathered}
\theta_{t}^{2}-\left|\nabla_{x} \theta\right|^{2}+\frac{\rho}{|\xi|}\left(\rho_{t}^{2}-\left|\nabla_{x} \rho\right|^{2}\right)=0, \\
\theta_{t} \rho_{t}-\nabla_{x} \theta \cdot \nabla_{x} \rho=0 .
\end{gathered}
$$

The function $\rho$ vanishes on the caustic surface $S_{\alpha}, \alpha=|\xi|^{-1}(\xi, \eta)$. For $\eta=0$, $S_{\alpha}=\partial \Omega$. As shown by Ludwig [24], one can prescribe $\theta$ on $S_{\alpha}$, as any solution of the associated surface eikonal equation, and then there is a unique solution to (3.6), (3.7), smooth up to $S_{\alpha}$, but the solution only exists on the illuminated side of the caustic. Thus $\rho$ and $\theta$ are defined on $\mathbf{R} \times\left(\mathbf{R}^{n} \backslash K\right)=\Omega$, only for $\eta<0$. However, as shown in [49], $\rho$ and $\theta$ may be continued smoothly to $\eta<0$ in such a fashion that (3.6), (3.7) is satisfied to infinite order on $\partial \Omega$ in this region, and this still implies that (3.5) yields a solution $\left(\bmod C^{\infty}\right)$ to the wave equation. Ludwig [24] also shows that we may choose the caustic surfaces in such a fashion that

$$
|\xi|^{-1} \rho=-|\xi|^{-1} \eta+0\left((|\eta| /|\xi|)^{\infty}\right) \text { on } \partial \Omega
$$

which is very convenient. An even stronger result follows from the work of Melrose [31], which we will discuss shortly.

Setting further terms equal to zero yields the following transport equations for $g$ and $h$; here $\nabla \theta$ stands for $\nabla_{(t, x)} \theta$, and the inner product is with respect to the Lorentz metric.

$$
\begin{aligned}
2 \nabla \theta \cdot \nabla g & +2 \frac{\rho}{|\xi|} \nabla \rho \cdot \nabla h+(\square \theta) g \\
& +\frac{\rho}{|\xi|}(\square \rho) h+\frac{1}{|\xi|}(\nabla \rho)^{2} h=-\square g,
\end{aligned}
$$




$$
2 \nabla \rho \cdot \nabla g+2 \nabla \theta \cdot \nabla h+(\square \rho) g+(\square \theta) h=-\square h
$$

where the equations are satisfied in the formal sense that equality holds for all terms of like degree of homogeniety. As for (3.6), (3.7), exact solutions on $\Omega$ exist for $\eta \leqslant 0$, which can be continued smoothly to $\eta>0$, as solutions to infinite order at $\partial \Omega$. Furthermore it can be arranged that

$$
h=0\left((|\eta| /|\xi|)^{\infty}\right) \text { on } \partial \Omega \text {. }
$$

We now show how $F$ can be determined so that the Dirichlet boundary condition (3.2) is satisfied. In fact, given (3.5), we have

$$
\begin{aligned}
\left.u\right|_{\partial \Omega} & =\iint\left[g+i h|\xi|^{-1 / 3} \frac{A^{\prime}\left(|\xi|^{-1 / 3} \rho\right)}{A\left(|\xi|^{-1 / 3} \rho\right)}\right] B e^{i(\theta+\gamma)} \hat{F} d \xi d \eta \\
& =J(F)
\end{aligned}
$$

where

$$
\gamma=\frac{2}{3}|\xi|\left[\left(\frac{\rho}{|\xi|}\right)^{3 / 2}-\left(-\frac{\eta}{|\xi|}\right)^{3 / 2}\right] \text { for } \eta<0 ; \gamma=0 \text { for } \eta>0,
$$

a smooth function (on $\partial \Omega$ ) homogeneous of degree 1 in $(\xi, \eta)$, and where

$$
B=\left\{\begin{array}{cc}
\frac{\Phi\left(|\xi|^{-1 / 3} \rho\right)}{\Phi\left(-|\xi|^{-1 / 3} \eta\right)} & \eta<0, \\
1, & \eta \geqslant 0,
\end{array}\right.
$$

so $B \in S_{1,0}^{0}$ on $\partial \Omega$. Since $h$ vanishes to infinite order on $\eta=0$, on $\partial \Omega$, it follows that the amplitude in (3.12) belongs to $S_{1,0}^{0}$. One can arrange that $g \neq 0$ near $\eta=0$, so $J$ is an elliptic Fourier integral operator, of classical type. The canonical transformation associated with $J$ maps the "characteristic variety," over which the grazing rays pass, to the variety $\eta=0$, and is locally bijective. Consequently, by the calculus of Fourier integral operators developed in [15], we can solve the equation $J(F)=f, \bmod C^{\infty}$, for $F$, and then the function $u$ given by (3.5) solves (3.1), (3.2), $\bmod C^{\infty}$.

The analysis of the singularities of $u$, given by (3.5), is an elaboration of the analysis given in \$2. For the details see Melrose [30] or Taylor [49]. The result is that $\operatorname{WF}(u)$ is contained in the set of rays passing over $\operatorname{WF}(f)$, going into positive time.

To solve the Neumann problem (3.1), (3.3), we again let $u$ be given by (3.5) and compute $\partial u /\left.\partial \nu\right|_{\partial \Omega}$. We have

$$
\begin{aligned}
\left.\frac{\partial}{\partial \nu} u\right|_{\partial \Omega}= & \iint\left[\rho_{\nu} g-\theta_{\nu} h+i h_{\nu}\right] C e^{i(\theta+\gamma)}|\xi|^{-1 / 3} \frac{A^{\prime}}{A}\left(|\xi|^{-1 / 3} \eta\right) \hat{F} d \xi d \eta \\
& +\iint\left[i \theta_{\nu} g+i|\xi|^{-1} \rho \rho_{\nu} h+g_{\nu}\right] B e^{i(\theta+\gamma)} \hat{F} d \xi d \eta \\
= & K_{1} Q F+K_{2} F
\end{aligned}
$$


where $C \in S_{1,0}^{0}$ is defined by

$$
\frac{A^{\prime}\left(|\xi|^{-1 / 3} \rho\right)}{A^{\prime}\left(-|\xi|^{-1 / 3} \eta\right)}=C e^{i \gamma},
$$

and $Q$ is a pseudo differential operator with symbol

$$
\sigma_{Q}=|\xi|^{-1 / 3} \frac{A^{\prime}}{A}\left(|\xi|^{-1 / 3} \eta\right) \in S_{1 / 3,0}^{0}
$$

We see that $K_{1}$ and $K_{2}$ are Fourier integral operators, with amplitudes in $S_{1,0}^{0}$. Also, the convexity of $\partial K$ implies that $\rho_{\nu} \neq 0$ at $\eta=0$ on $\partial \Omega$, while $\theta_{\nu}=0$. It follows that $K_{1}$ is elliptic. Also, $J, K_{1}$, and $K_{2}$ are all associated with the same canonical transformation. It follows that, denoting by $J^{-1}$ a microlocal parametrix for $J$,

$$
J^{-1}\left(K_{1} Q+K_{2}\right)=A Q+B
$$

where $A \in \mathrm{OP} S_{1,0}^{0}$ is elliptic and $B \in \mathrm{OP} S_{1,0}^{1}$ has principal symbol vanishing at $\eta=0$.

If $N$ denotes the Neumann operator $g=N f$, where $g=\partial u /\left.\partial \nu\right|_{\partial \Omega}$ for $u$ solving (3.1), (3.2), $u=0$ for $t \ll 0$, we see from (3.13) and (3.15) that, near grazing directions,

$$
N=J(A Q+B) J^{-1}
$$

This formula plays a crucial role in the application of the grazing ray analysis to problems in scattering theory, as we'll see in the next section, but now we use it to solve (3.1), (3.3), mod $C^{\infty}$, thus obtaining the same information on propagation of singularities near grazing rays for Neumann boundary conditions as we obtained for Dirichlet boundary conditions.

Note that $Q^{-1} \in \mathrm{OP} S_{1 / 3,0}^{1 / 3}$, since neither $A(s)$ nor $A^{\prime}(s)$ has any real zeros. Now writing $A Q+B=\left(\mathrm{A}+B Q^{-1}\right) Q$, we see that the principal symbol of $B Q^{-1}$ is of the form (with $b_{0} \in S_{1,0}^{0}$ )

$|\xi|^{1 / 3} \eta \frac{A}{A^{\prime}}\left(|\xi|^{-1 / 3} \eta\right) b_{0}(t, x, \xi, \eta)=|\xi|^{2 / 3}|\xi|^{-1 / 3} \eta \frac{A}{A^{\prime}}\left(|\xi|^{-1 / 3} \eta\right) b_{0}(t, x, \xi, \eta)$.

This belongs to $S_{1 / 3,0}^{1}$ and is small in a small conic neighborhood of $\eta=0$. Consequently $A+B Q^{-1} \in \mathrm{OP} S_{1 / 3,0}^{1}$ is elliptic, so $\left(A+B Q^{-1}\right)^{-1} \in$ OP $S_{1 / 3,0}^{-1}$. Consequently we can write

$$
N^{-1}=J Q^{-1}\left(A+B Q^{-1}\right)^{-1} J^{-1} \text {. }
$$

Thus we can write down a solution to (3.1), (3.3) as merely the solution we've already constructed to (3.1), (3.2), setting $f=N^{-1} g=J Q^{-1}(A+$ $\left.B Q^{-1}\right)^{-1} J^{-1} g$.

A large variety of boundary conditions for hyperbolic equations is treatable by the method outlined above. In [50] the diffraction problem is treated for Maxwell's equations for electromagnetic waves on the exterior of a smooth, convex, perfectly conducting obstacle; this leads to a nonelliptic (but hypoelliptic) Fourier integral equation similar to $\left(K_{1} Q+K_{2}\right) F=g$. Also the diffraction problem for a clear convex obstacle, of index of refraction different from 1, is treated; this leads to an elliptic Fourier integral equation, 
but one more complicated than $J F=f$, since the operator $Q$ is also involved. Generalizing the Dirichlet and transmission problems, [50] also treats the diffraction problem for first order systems satisfying the Kreiss condition (micro-locally). There is an error at one point in this argument, which is corrected in [51]; in [51] there is also a treatment of the diffraction problem for a general class of systems for which one has a loss of $1 / 3$ derivative when solving the appropriate hypoelliptic Fourier integral equation; this class is related to the class of Kreiss-well-posed problems as the Neumann boundary condition is related to the Dirichlet boundary condition.

There is a slightly different approach to the diffraction problem, due to Melrose, which follows from the work [31] of Melrose on the equivalence of glancing hypersurfaces. We say two hypersurfaces of $T^{*}(\Omega)$, given by $F=\{f$ $=0\}$ and $G=\{g=0\}$ are glancing at $z \in F \cap G$ if $d f(z)$ and $d g(z)$ are linearly independent, and letting $\{f, g\}=H_{f} g$,

$$
\begin{gathered}
\{f, g\}(z)=0, \\
\{f,\{f, g\}\}(z) \neq 0 \text { and }\{g,\{g, f\}\}(z) \neq 0 .
\end{gathered}
$$

In [31] it is shown that any two pairs of glancing hypersurfaces, $F, G$ and $F^{\prime}$, $G^{\prime}$ (glancing at $z^{\prime}$ ) are locally equivalent in the sense that there is a symplectic map taking $z$ to $z^{\prime}$ and (locally) $F$ to $F^{\prime}, G$ to $G^{\prime}$. If the hypersurfaces are conic and we assume that $d f(z), d g(z)$ and $\Sigma \xi_{j} d x_{j}$ are linearly independent at $z$, with a similar assumption at $z^{\prime}$, then the symplectic map can be chosen to be homogeneous of degree 1 . The solution to this problem answers affirmatively, in the $C^{\infty}$ category, a conjecture of Sato (which has been shown to be false in the real analytic category). The solution to this equivalence problem allows one to reduce the study of the phase functions and amplitudes occurring in the parametrix to the construction made in the example studied by Friedlander [13]. The solution to this equivalence problem has also enabled Melrose [33] and Andersson and Melrose [1] to analyze propagation of singularities along the boundary on the interior of a convex region.

Finally, we refer the reader to the papers of Eskin [11], [12], dealing with these propagation of singularities problems.

4. Diffraction theory and scattering theory. A great deal of classical scattering theory is devoted to the study of the scattering amplitude associated with an obstacle $K$, which we now discuss. We will restrict our attention to Dirichlet boundary conditions, though modifications of these methods handle many other boundary conditions, such as the Neumann condition and the boundary value problem for electromagnetic waves on the exterior of a perfectly conducting obstacle; see [25], [27]. We assume the obstacle $K$ is smooth and bounded and strictly convex, $K \subset \mathbf{R}^{3}$. Pick unit vectors $\theta, \omega$ in $\mathbf{R}^{3}$, and let $\lambda \in \mathbf{R}$.

Let $u_{s}(x, \omega, \lambda)$ be the unique solution to the exterior boundary value problem

$$
\begin{array}{r}
\left(\Delta+\lambda^{2}\right) u_{s}=0, \\
\left.u_{s}\right|_{\partial K}=-e^{-i \lambda x \cdot \omega}
\end{array}
$$


satisfying the "radiation condition" at infinity. Such a solution has the asymptotic behavior $u_{s}(r \theta) \sim\left(e^{i \lambda r} / r\right) b(\theta)+o(1 / r)$, and the scattering amplitude $a_{s}$ is defined by the formula

$$
a_{s}(\theta, \omega, \lambda)=\lim _{r \rightarrow \infty} r e^{-i \lambda} u_{s}(r \theta, \omega, \lambda) .
$$

The solution $u_{s}$ to (4.1), (4.2) can be represented by Green's formula:

$$
u_{s}(x)=\int_{\partial K}\left(u_{s}(y) \frac{\partial}{\partial \nu} G_{\lambda}(x-y)-\frac{\partial u_{s}}{\partial \nu} G_{\lambda}(x-y)\right) d S(y)
$$

where $G_{\lambda}(x)=e^{i \lambda|x|} /|x|$. Applying (4.3) yields

$$
a_{s}(\theta, \omega, \lambda)=\int_{\partial K} e^{-i \lambda \theta \cdot y}\left[i \lambda(\nu \cdot \theta) u_{s}(y, \omega, \lambda)+\frac{\partial}{\partial \nu} u_{s}(y, \omega, \lambda)\right] d S(y) .
$$

A more convenient representation is obtained by taking the Fourier transform with respect to $\lambda$. We have

$$
\hat{a}_{s}(\theta, \omega, t)=\int_{\partial K}\left(\frac{\partial}{\partial \nu}-(\nu \cdot \theta) \frac{\partial}{\nu t}\right) \hat{u}_{s}(y, \omega, t+y \cdot \theta) d S(y)
$$

where $\hat{u}_{s}(y, \omega, t)$ is characterized in the following fashion. It solves the boundary value problem

$$
\begin{gathered}
\left(\frac{\partial^{2}}{\partial t^{2}}-\Delta\right) \hat{u}_{s}=0, \\
\left.\hat{u}_{s}\right|_{\mathrm{R} \times \partial K}=-\left.\delta(t-y \cdot \omega)\right|_{\mathbf{R} \times \partial K}
\end{gathered}
$$

and is "outgoing," i.e. $\hat{u}_{s} \equiv 0$ for $t \ll 0$. We replace (4.5) by

$$
\begin{gathered}
a_{s}(\theta, \omega, \lambda)=\int_{-\infty}^{\infty} \int_{\partial K} e^{-i \lambda t}\left(\frac{\partial}{\partial \nu}-(\nu \cdot \theta) \frac{\partial}{\partial t}\right) \hat{u}_{s}(y, \omega, t+y \cdot \theta) d S(y) d t \\
=\int_{-\infty}^{\infty} \int_{\partial K} e^{-i \lambda t}\left(N-(\nu \cdot \theta) \frac{\partial}{\partial t}\right) \delta(t+y \cdot \theta-y \cdot \omega) d S(y) d t
\end{gathered}
$$

where $N$ is the Neumann operator discussed in the previous section; recall (3.16).

Now these formulas are standard, but until the Neumann operator was analyzed it was not possible to use them to give a rigorous analysis of the scattering amplitude $a_{s}$ as $\lambda \rightarrow \infty$. Calculations were made based on the Kirchoff approximation, which states that the normal derivative of $u_{s}$ is given by

$$
\begin{aligned}
& \frac{\partial u_{s}}{\partial \nu} \approx-\nu \cdot \omega(i \lambda) e^{-i \lambda x \cdot \omega} \quad \text { on } \partial K^{+}, \\
& \frac{\partial u_{s}}{\partial \nu} \approx \nu \cdot \omega(i \lambda) e^{-i \lambda x \cdot \omega} \quad \text { on } \partial K^{-}
\end{aligned}
$$

where $\partial K^{+}=\{x \in \partial K: \nu \cdot \omega>0\}$ is the illuminated side of $\partial K$ and $\partial K^{-}=$ $\{x \in \partial K: \nu \cdot \omega<0\}$ is the shadow side. This rule was motivated by the idea that the scattered field, for large $\lambda$, is approximately given by the rules of 
geometrical optics. With the solution to the grazing ray problem we can say that (4.9) is valid, to first order, as $\lambda \rightarrow \infty$, at least away from the grazing submanifold $G(\omega)=\{x \in \partial K: v \cdot \omega=0\}$. There the formula (3.16) provides a more serious correction. Since $G(\omega)$ is a small set, one might expect that integral formulas such as (4.8) will have the same asymptotic behavior, in the principal part, as the expression one would get by replacing $N \delta$ by its Kirchoff approximation. This is correct, though the proof is not always easy.

Analyzing the asymptotic behavior of $a_{s}(\theta, \omega, \lambda)$ as $\lambda \rightarrow \infty$, for fixed $\theta, \omega$, is equivalent to analyzing the singularities of $\hat{a}_{s}(\theta, \omega, t)$. First we locate the singularities of

$$
\hat{a}_{s}(\theta, \omega, t)=\int_{\partial K} G(y, \omega, t+y \cdot \theta) d S(y)
$$

where $G(y, \omega, t)=(N-(\nu \cdot \theta) \partial / \partial t) \delta(y \cdot \omega-t)$. From (3.16) we see that $N$ is pseudo-local, i.e. preserves wave front sets. Consequently $\operatorname{WF}(G) \subset$ $\operatorname{WF}(\delta)$; thus $\operatorname{WF}(G)$ is contained in the set of normals in $\mathbf{R} \times \partial K$ to the hypersurface $t=y \cdot \omega$. From general results on wave front sets (see [7] or [15]) it follows that $\hat{a}_{s}(\theta, \omega, t)$ is only singular for those values $t_{0}$ for which the hypersurface $t=y \cdot \omega$ intersects the hypersurface $t=t_{0}+y \cdot \theta$, nontransversally. For $\theta=\omega$ this happens precisely at $t_{0}=0$, where the two hypersurfaces coincide exactly; this "big" singularity in $\hat{a}_{s}(\theta, \theta, t)$ leads to the "diffraction peak" in the forward scattering amplitude $a_{s}(\theta, \theta, \lambda)$. For each pair $\theta, \omega$ with $\theta \neq \omega$, there are precisely two such values of $t$, given by the min and max, respectively, of $y \cdot(\theta-\omega), y \in \partial K$. At these two points, $y_{1}(\theta, \omega)$ and $y_{2}(\theta, \omega)$, the normals to $\partial K$ are parallel (resp. antiparallel) to $\theta-\omega$. One can show that in fact there is no singularity at $t=\max _{y \in \partial K} y \cdot(\theta-\omega)$.

To analyze the singularity of $\hat{a}_{s}(\theta, \omega, t)$ when $\theta \neq \omega$, one need only work near $y=y_{1}(\theta, \omega)$. By means of the method of stationary phase, (4.8) can be asymptotically evaluated. See [25] or [27]. One obtains, for the Dirichlet problem,

$$
a_{s}(\theta, \omega, \lambda)=\mathscr{K}\left(y_{1}\right)^{-1 / 2} e^{-i \lambda y_{1} \cdot(\theta-\omega)} \frac{\nu \cdot \theta}{|\theta-\omega|}+O\left(\lambda^{-1}\right)
$$

where $\mathcal{K}\left(y_{1}\right)$ is the curvature of $\partial K$ at $y_{1}(\theta, \omega)$. This formula has several interesting implications. In particular, given a knowledge of the asymptotic behavior of $\left|a_{s}(\theta, \omega, \lambda)\right|$ for sufficiently many $(\theta, \omega)$, one can determine the curvature of $\partial K$ in every direction, and this uniquely determines $K$. Similar work done in [27] on clear convex obstacles enables one to determine not only the shape of an obstacle, but also its index of refraction, at the boundary, given the asymptotic behavior of the "filtered scattering amplitude."

The analysis of the forward scattering amplitude $a_{s}(\theta, \theta, \lambda)$, carried out in [28], involves greater difficulties, since in this case the grazing submanifold $G(\omega)$ makes a bigger contribution to the asymptotic behavior, and certain unpleasant features of $N$ have to be dealt with. The Kirchoff approximation suggests that

$$
a_{s}(\theta, \theta, \lambda) \sim 2 i \lambda \int_{\partial K^{+}} \nu(x) \cdot \theta d S(x)=2 i \lambda \operatorname{Area}(\delta(\partial K, \theta))
$$


where $\mathcal{S}(\partial K, \theta)$ is the projection of $\partial K$ onto the plane orthogonal to $\theta$, the "shadow projection" of $\partial K$ in the direction $\theta$. In this case, one cannot obtain the asymptotic behavior by looking at (4.8) and applying the stationary phase method in a straightforward manner, due to the fact that symbols in $S_{1 / 3,0}^{0}$ are involved in the formula (3.16) for the Neumann operator $N$.

We analyze

$$
\int_{-\infty}^{\infty} \int_{\partial K} e^{-i \lambda t} N \delta(t+y \cdot \theta-y \cdot \omega) d S(y) d t=\left\langle e^{-i \lambda t}, J(A Q+B) J^{-1} \delta\right\rangle
$$

as follows. Write it as

$$
\left\langle J^{*}\left(e^{-i \lambda t}\right),(A Q+B) J^{-1} \delta\right\rangle .
$$

As is shown in [28], the canonical transformation associated with $J^{-1}$ can be arranged to yield no caustics when applied to $\operatorname{WF}(\delta)$, and consequently, using the stationary phase method, one obtains an asymptotic progressing wave expansion

$$
J^{-1} \delta \sim B_{0} \delta(\tilde{\psi})+B_{1} \delta(\tilde{\psi})+\cdots
$$

with a certain phase function $\tilde{\psi}$, and $B_{j} \in$ OP $S^{-j}$ classical pseudo differential operators. Also we get the asymptotic expansion

$$
J^{*}\left(e^{-i \lambda t}\right) \sim e^{-i \lambda \tilde{\psi}}\left(a_{0}+a_{1} \lambda^{-1}+\cdots\right) .
$$

Consequently, (4.12) becomes

$$
\left\langle a_{0} e^{-i \lambda \tilde{\psi}},(A Q+B) B_{0} \delta(\tilde{\psi})\right\rangle+\cdots
$$

In order to compare this with the integral we would get by replacing $N \delta$ by its Kirchoff approximation, we split $A Q+B$ into two parts. Introduce the cut-offs $\varphi_{1}(\xi, \eta)=\varphi\left(|\xi|^{-a} \eta\right) \in S_{a, 0}^{0}$ and $\varphi_{2}(\xi, \eta)=1-\varphi_{1}(\xi, \eta)$, with $\varphi \in$ $C_{0}^{\infty}(\mathbf{R}), \varphi(s)=1$ for $|s|<1$, choosing $a$ such that $\frac{1}{2}<a<1$. Then write (4.12) as

$$
\left\langle J^{*}\left(e^{-i \lambda t}\right),\left(A Q \varphi_{2}(D)+B\right) J^{-1} \delta\right\rangle+\left\langle J^{*}\left(e^{-i \lambda t}\right), A Q \varphi_{1}(D) J^{-1} \delta\right\rangle .
$$

The first term can be asymptotically evaluated by the stationary phase method, since $A Q \varphi_{2}(D)+B \in \mathrm{OP} S_{a, 0}^{1}$, and one gets agreement with the Kirchoff approximation. It remains only to estimate the error term

$$
\left\langle J^{*}\left(e^{-i \lambda t}\right), A Q \varphi_{1}(D)\left(J^{-1} \delta\right)\right\rangle .
$$

Now $A Q \varphi_{1}(D) \in \mathrm{OP} S_{1 / 3,0}^{1 / 2+a / 2}$, so has order less than 1 . Consequently one expects (4.16) to grow more slowly as $\lambda \rightarrow \infty$ than the principal terms. However, it is still not possible to get a simple estimate of (4.16) by the stationary phase method, since the type $\left(\frac{1}{3}, 0\right)$ is not amenable to such analysis.

Fortunately, the special structure of the operator $A Q \varphi_{1}(D)$ leads to $L^{p}$ estimates which yield an adequate estimate of (4.16). In fact,

$$
A Q \varphi_{1}(D): W_{p}^{s} \rightarrow W_{p}^{s-(1 / 2+a / 2)}, \quad 1<p<\infty .
$$

Since (4.13) implies that $J^{-1} \delta \in W_{1+\varepsilon^{\prime}}^{-\varepsilon}$, using (4.14) we easily obtain 


$$
\left|\left\langle J^{*}\left(e^{-i \lambda t}\right), A Q \varphi_{1}(D)\left(J^{-1} \delta\right)\right\rangle\right| \leqslant c \lambda^{1 / 2+a / 2+\varepsilon} .
$$

In [28], taking $a=3 / 5$, and authors deduced that

$$
a_{s}(\theta, \theta, \lambda)=2 i \lambda \text { Area }(\delta(\partial K, \theta))+O\left(\lambda^{4 / 5+\varepsilon}\right) .
$$

With very little extra work one can improve the error estimate to $O\left(\lambda^{3 / 4+\varepsilon}\right)$, as indicated in [47, Chapter IV]. With a bit more work one can improve the error estimate to $O\left(\lambda^{1 / 3+\varepsilon}\right)$, and it seems that one can produce the sharp error estimate $O\left(\lambda^{1 / 3}\right)$, though the details have not yet been worked out.

(4.17) is a special case of the following result. Define the class of symbols $\mathfrak{N}_{\rho}^{m}$ by the condition $p(x, \xi) \in \mathfrak{N}_{\rho}^{m}$ if and only if, for all multi-indices $\alpha$,

$$
\xi^{\alpha} D_{\xi}^{\alpha} p(x, \xi) \in S_{\rho, 0}^{m} \text {. }
$$

We say $p(x, D) \in \mathrm{OP} \mathfrak{T}_{\rho}^{m}$. Using the Marcinkiewicz multiplier theorem (Theorem 6', Chapter IV of Stein [45]), one can deduce that

$$
p(x, D) \in \mathrm{OP} \Re_{\rho}^{m} \Rightarrow p(x, D): W_{p}^{s} \rightarrow W_{p}^{s-m}, \quad 1<p<\infty .
$$

It is not hard to show that $A Q \varphi_{1}(D) \in \mathrm{OP} \Re_{1 / 3}^{1 / 2+a / 2}$.

It is still an open problem to obtain an asymptotic expansion for $a_{s}(\theta, \omega, \lambda)$ that is uniformly valid for $\theta$ near $\omega$.

\section{REFERENCES}

1. K. Andersson and R. Melrose, The propagation of singularities along gliding rays, Sem. Goulaoic-Schwartz 1976-1977, Exposé No. 1.

2. G. Birkhoff, Quantum mechanics and asymptotic series, Bull. Amer. Math. Soc. 39 (1933), 681-700.

3. J. Bony and P. Shapira, Propagation des singularités analytiques pour les solutions des equations differentielles, Ann. Inst. Fourier 26 (1976), 81-140.

4. J. Chazarain, Propagation des singularities pour une classe d'opérateurs a caractéristiques multiples et résobulité locale, Ann. Inst. Fourier (Grenoble) 24 (1974), 203-223.

5. Construction de la paramétrix du problème mixte hyperbolique pour l'equation des ordres, C. R. Acad. Sci. Paris 276 (1973), 1213-1215.

6. R. Courant and P. Lax, The propagation of discontinuities in wave motion, Proc. Nat. Acad. Sci. 42 (1956), 872-876.

7. J. Duistermaat, Fourier integral operators, Courant Inst. of Math. Sci. New York University, Lecture Notes, 1973.

8. J. Duistermaat and L. Hörmander, Fourier integral operators. II, Acta Math. 128 (1972), 183-259.

9. Yu. V. Egorov, On canonical transformations of pseudo-differential operators, Uspehi Mat. Nauk. 24 (1969), 235-236.

10. A. Erdélyi, Asymptotic expansions, Dover, New York, 1965.

11. G. Eskin, A parametrix for mixed problems for strictly hyperbolic equations of arbitrary order, Comm. Partial Differential Equations 1 (1976), 521-560.

12. Propagation of singularities for interior mixed hyperbolic problem, Sem. GoulaoicSchwartz 1976-1977, Exposé No. 12.

13. F. G. Friedlander, The wave front set of the solution of a simple initial boundary value problem with glancing rays, Proc. Cambridge Philos. Soc. 79 (1976), 145-159.

14. F. G. Friedlander and R. Melrose, The wave front set of the solution of a simple initial boundary value problem with glancing rays. II, Proc. Cambridge Philos. Soc. 81 (1977), 97-120.

15. L. Hörmander, Fourier integral operators. I, Acta Math. 127 (1971), 79-183.

16. On the existence and regularity of solutions of linear partial differential equations, Enseignement Math. 17 (1971), 99-163.

17. , Pseudo-differential operators and hypoelliptic equations, Singular Integrals, Proc.

Sympos. Pure Math., vol. 10, Amer. Math. Soc., Providence, R. I., 1967, pp. 138-183. 
18. Uniqueness theorems and wave front sets for solutions of linear differential equations with analytic coefficients, Comm. Pure Appl. Math. 24 (1971), 671-703.

19. J. Keller, Diffraction by a convex cylinder, Trans. I. R. E. AP-4 (1956), 312-321.

20. H. Kreiss, Initial boundary value problems for hyperbolic systems, Comm. Pure Appl. Math. 23 (1970), 277-298.

21. P. Lax, Asymptotic solutions of oscillatory initial value problems, Duke Math. J. 24 (1957), 627-646.

22. P. Lax and R. Phillips, Scattering theory, Academic Press, New York, 1967.

23. D. Ludwig, Uniform asymptotic expansions at a caustic, Comm. Pure Appl. Math. 19 (1966), 215-250.

24. __ Uniform asymptotic expansions of the field scattered by a convex object at high frequencies, Comm. Pure Appl. Math. 19 (1967), 103-138.

25. A. Majda, High frequency asymptotics for the scattering matrix and the inverse problem of acoustical scattering, Comm. Pure Appl. Math. 29 (1976), 261-291.

26. A. Majda and S. Osher, Reflection of singularities at the boundary, Comm. Pure Appl. Math. 28 (1975), 479-499.

27. A. Majda and M. Taylor, Inverse scattering problems for transparent obstacles, electromagnetic waves, and hyperbolic systems, Comm. Partial Differential Equations 2 (1977).

28. _ The asymptotic behavior of the diffraction peak in classical scattering, Comm. Pure Appl. Math. 30 (1977), 639-669.

29. R. Melrose, Local Fourier-Airy integral operators, Duke Math. J. 42 (1975), 583-604.

30. __ Microlocal parametrices for diffractive boundary value problems, Duke Math. J. 42 (1975), 605-635.

31. __ Equivalence of glancing hypersurfaces, Invent. Math. 37 (1976), 165-191.

32. Normal self-intersections of the characteristic variety, Bull. Amer. Math. Soc. 81 (1975), 939-940.

33. R. Melrose and K. G. Andersson, The propagation of singularities along gliding rays, Invent. Math. 41 (1977), 197-232.

34. C. Morawetz, Exponential decay of solutions of the wave equation, Comm. Pure Appl. Math. 19 (1966), 439-444.

35. C. Morawetz and D. Ludwig, The generalized Huyghens' principle for reflecting bodies, Comm. Pure Appl. Math. 22 (1969), 189-205.

36. L. Nirenberg, Lectures on linear partial differential equations, CBMS Regional Conf. Ser. in Math., no. 17, Amer. Math. Soc., Providence, R. I., 1973.

37. J. Polking, Boundary value problems for parabolic systems of partial differential equations, Proc. Sympos. Pure Math., vol. 10, Amer. Math. Soc., Providence, R. I., 1967, pp. 243-274.

38. A. Povzner and K. Suharevskii, Discontinuities of the Green's function of a mixed problem for the wave equation, Mat. Sb. 51 (1960), 3-26; Amer. Math. Soc. Transl. (2) 47 (1965), pp. 131-156.

39. J. Ralston, Solutions of the wave equation with localized energy, Comm. Pure Appl. Math. 22 (1969), 807-824.

40. Lord Rayleigh, On waves propagated along the plane surface of an elastic solid, Proc. London Math. Soc. 17 (1885), 4-11.

41. R. Sakamoto, Mixed problems for hyperbolic equations. I, II, J. Math. Kyoto Univ. 10 (1970), 349-373; 403-417.

42. M. Sato, M. Kashiwara and T. Kawai, Hyperfunctions and pseudo differential equations, Lecture Notes in Math., vol. 287, Springer-Verlag, Berlin and New York, 1973, pp. 265-529.

43. J. Sjöstrand, Propagation of singularities for operators with multiple involutive characteristics, Ann. Inst. Fourier (Grenoble) 26 (1976), 141-155.

44. A. Sommerfeld and J. Runge, Anwendung der Vektorrechnung auf die Grundlagen der geometrischen Optik, Ann. Phys. 35 (1911), 277-298.

45. E. Stein, Singular integrals and differentiability properties of functions, Princeton Univ. Press, Princeton, N. J., 1970.

46. M. Taylor, Pseudo differential operators, Lecture Notes in Math., vol.416, Springer-Verlag, Berlin and New York, 1974.

47. ___ Pseudo differential operators. Part II (to appear). 
48. Reflection of singularities of solutions to systems of differential equations, Comm. Pure Appl. Math. 28 (1975), 457-478.

49. - Grazing rays and reflection of singularities of solutions to wave equations, Comm. Pure Appl. Math. 29 (1976), 1-38.

50. (systems) Comm. Pure Appl. Math. 29 (1976), 463-481.

51. __ Grazing rays and reflection of singularities of solutions to systems of wave equations. Part III (to appear).

52. __ Rayleigh waves in linear elasticity as a propagation of singularities phenomenon, Park City Conf. in PDE and Geometry, 1977 (to appear).

Department of Mathematics, Rice University, Houston, TeXas 77001 